\title{
Agôn
}

Revue des arts de la scène

$5 \mid 2012$

L'entrée en scène

\section{Entrer dehors : faire et défaire son entrée dans les théâtres de rue}

\section{Géraldine Prévot}

\section{(2) OpenEdition}

Journals

Édition électronique

URL : http://journals.openedition.org/agon/2441

DOI : 10.4000/agon.2441

ISSN : 1961-8581

\section{Éditeur}

Association Agôn

\section{Référence électronique}

Géraldine Prévot, «Entrer dehors : faire et défaire son entrée dans les théâtres de rue », Agôn [En ligne], 5 | 2012, mis en ligne le 21 janvier 2013, consulté le 02 mai 2019. URL : http:// journals.openedition.org/agon/2441; DOI : 10.4000/agon.2441

Ce document a été généré automatiquement le 2 mai 2019.

Association Agôn et les auteurs des articles 


\title{
Entrer dehors : faire et défaire son entrée dans les théâtres de rue
}

\author{
Géraldine Prévot
}

Je vous construirai une ville avec des loques, moi!

Henri Michaux, Contre!

1 L'entrée en scène est un vecteur de signes : il s'agit pour le comédien de faire entrer des formes signifiantes dans un lieu qui, jusque là, passait pour inerte et inoccupé. En faisant son entrée, le comédien entre dans le domaine du visible. D'ores et déjà, on peut proposer de l'entrée en scène deux approches: l'une concrète, l'autre plus conceptuelle. La première repose sur le passage $d u$ comédien $d u$ hors-scène à la scène, sur le franchissement d'un seuil, qui peut exister de différentes manières. Si l'on en croit la définition courante de l'entrée que l'on trouve dans les dictionnaires, elle réside en une « action d'entrer, de passer de l'extérieur à l'intérieur ». Dans le cas de l'entrée en scène, cet intérieur serait la scène, supposément bien délimitée et enclose dans la salle de théâtre, et qui est également le lieu de la visibilité, le lieu où l'on se rend visible aux spectateurs : l'entrée en scène se double d'une entrée en jeu et s'y superpose. Mais qu'en est-il quand il s'agit d'entrer dehors ? La question du seuil se pose alors de manière tout à fait différente. Nous nous proposons ici d'étudier ce protocole liminaire de la représentation théâtrale dans le contexte du théâtre de rue, qui précisément remet en cause la définition de la scène comme espace séparé.

2 La deuxième approche, plus conceptuelle, interroge la nature même de l'acte théâtral à venir. L'entrée en scène est une forme de prospection; dans le lieu théâtral, elle éveille les potentialités $d u$ spectacle à venir. Les diverses formes d'entrées en scène métaphorisent, ou plutôt métonymisent, les diverses formes de représentations théâtrales. Qu'elle soit escamotée, spectaculaire, reportée, diluée, l'entrée en scène dit quelque chose du spectacle à venir. Elle est une forme de naissance du théâtre, au point qu'Antoine Vitez affirmait que «manquer son entrée, c'est mourir... ${ }^{1}$; proprement essentielle au comédien et au spectacle à venir, l'entrée en scène scellerait la «vie » de l'acteur et de la représentation, devenant par là même une entrée en jeu. L'entrée en 
scène a un espace et un temps, ou plutôt, c'est elle qui régit et active ces coordonnées de la représentation.

3 Or, dans le cadre des arts de la rue, l'espace-temps de l'entrée en scène est assez singulier puisqu'il est souvent extrêmement mobile. Nous nous demanderons ici comment la fluctuation même des espaces scéniques permet de questionner la polysémie de l'entrée en scène, ainsi que sa dimension anthropologique dans le contexte des arts de la rue. Les théâtres de rue semblent en effet redoubler la dimension rituelle inhérente à toute forme théâtrale ${ }^{2}$, et l'entrée en scène est précisément le moment où se cristallise le questionnement de ces formes théâtrales comme rites de passage. Nous essaierons ici, en reprenant tout au long de l'étude des paramètres élaborés par Anne Gonon ${ }^{3}$ (confinement/ouverture, mobilité/immobilité, acteurs/assistance), de dresser une esquisse de typologie des entrées en scène dans le théâtre de rue: de l'apparition à l'effraction, l'entrée en scène dans les arts de la rue propose un questionnement tant anthropologique qu'esthétique de la notion de seuil.

Notre réflexion, qui sera illustrée d'exemples tirés, sauf mention contraire, de spectacles créés dans le cadre du Festival international de théâtre de rue d'Aurillac en 2011 et $2012^{4}$, essaiera de spécifier certaines modalités des entrées en scène dans ces théâtres en extérieur, et de proposer une typologie raisonnée de ces dispositifs.

\section{Spatialité de l'entrée en scène : vers une typologie des espaces}

5 Il ne va pas de soi que le théâtre de rue fasse disparaître la scène; toutefois, les délimitations scéniques sont très souvent un support de jeu, voire le support principal, et sont fréquemment mouvantes. Avant d'approfondir la polysémie de l'entrée en scène, nous nous proposons d'étudier ses espaces.

En effet, au vu de la diversité des spectacles de théâtre de rue et des formes d'entrées en scène qu'ils proposent, il semble nécessaire de dresser une typologie, forcément lacunaire, des espaces de jeu, tant ils sont pour le spectateur le premier indice pour essayer de comprendre d'où va surgir le spectacle. En effet, l'entrée en scène de l'acteur de rue consiste à faire exister comme il l'entend un espace qui n'est pas initialement prévu pour le théâtre; dans une certaine mesure, la scène fait son entrée avec le comédien. De manière presque systématique, l'acteur de rue entre par effraction dans un espace qui lui est interdit : son entrée est un passage en force, et il convient donc, avant d'envisager plus précisément la nature et la diversité de ces entrées, de se pencher sur ces espaces scéniques fortuits. Nous nous proposons ici d'étudier trois espaces paradigmatiques des théâtres de rue, qui tous peuvent s'inscrire dans le champ du " théâtre environnemental ${ }^{5}$ ", tel que le définit Richard Schechner, qui utilise aussi le terme d'« espace total ». Sans désigner uniquement des espaces extérieurs, ce terme peut s'appliquer aux spectacles étudiés ici :

Le théâtre environnemental encourage les échanges à travers un espace organisé dans son ensemble et dans lequel les aires occupées par le public s'assimilent à une mer traversée à la nage par les acteurs, et les aires de jeu à des îles ou des continents bordées par le public. [...] L'utilisation de l'espace dans le théâtre environnemental repose fondamentalement sur la collaboration. 
7 Ce critère de la « collaboration » répond à celui d' « ouverture » proposé par Anne Gonon, qu'elle reprend à Denis Guénoun, et qui vient compléter notre tentative de définition d'une spatialité propre aux théâtres de rue.

Par une technique de surimpression, le spectateur voit simultanément deux choses : ce qui est fictif posé sur ce qui est réel, ce qui change et varie posé sur ce qui se maintient. [...] [Cette] double vue est métaphysique et politique. Elle fracture la réalité, c'est une disruption qui affirme la possibilité d'un espace ouvert ${ }^{6}$.

8 La «disruption » que décrit Denis Guénoun s'inscrit pleinement dans le questionnement de l'entrée en scène dans l'espace urbain, puisqu'elle permet de l'étudier comme une rupture dans le continuum social ; ainsi, étudier les différents espaces, en esquisser une typologie, permettra d'envisager les différentes formes que peut revêtir cette disruption.

9 L'espace « naturel » retiendra d'abord notre attention : il questionne l'entrée en scène de manière spécifique, notamment par son caractère a priori illimité. Ensuite, l'espace urbain de la déambulation nous permettra d'étudier des formes théâtrales qui proposent, dans une certaine mesure, une entrée en scène perpétuelle. Enfin, certains espaces de théâtre de rue proposent des scènes multiples, mobiles, qui interrogent très fortement l'interaction entre l'entrée en scène et la disposition des spectateurs.

\section{L'espace naturel, ou comment entrer dehors}

10 Certains spectacles dits de "théâtre de rue » ne se passent précisément pas dans la rue, au sens strict, mais dans des espaces péri-urbains, marqués par une omniprésence de la nature. C'est le cas du spectacle Pleine forêt sensible, proposé par la compagnie Les Souffleurs, qui a lieu dans une clairière au milieu d'une forêt, où les comédiens, déjà installés dans l'espace de jeu, attendent l'arrivée des spectateurs. Dans ce spectacle, les comédiens ne font pas leur entrée mais se fondent, formellement, dans l'espace naturel : juchés sur des promontoires dont la verticalité s'inscrit dans la géométrie d'ensemble de la forêt, ils constituent un écho incarné aux arbres environnants. L'entrée en scène dans ce type d'espace suppose une prise en compte de ses spécificités: ici, le silence de la clairière, son obscurité, sa circularité, répondent parfaitement à la démarche d'ensemble du spectacle, qui consiste à souffler des poèmes aux oreilles des spectateurs, à l'aide de tubes résonants.

11 Mais ce spectacle n'épuise pas, bien au contraire, les modalités de l'entrée en scène dans ce type d'espace naturel, puisqu'il choisit d'escamoter celle des comédiens, au profit d'une seule apparition, nous y reviendrons. Le groupe ZUR ${ }^{7}$, par contre, utilise pleinement l'immensité et l'irrégularité naturelles dans son spectacle HoriZ曷e Km zéro ${ }^{8}$, dans lequel il propose une série d'entrées dehors. Deux points peuvent être abordés: le jeu avec les limites de l'espace naturel ; le rôle des spectateurs dans cet espace naturel.

12 En effet, dans ce spectacle, le groupe ZUR travaille pleinement les présupposés du paysage choisi pour présenter le spectacle, afin de jouer avec les perceptions des spectateurs. Les différentes entrées en scène fonctionnent de manière extrêmement concomitante à un travail de la lumière qui vise à remettre en jeu les perceptions des spectateurs sur le paysage dans lequel ils se trouvent. Le spectacle a lieu de nuit, et les différentes entrées des comédiens sont autant de sources lumineuses (feu, torches, lanternes, projection d'images...) qui s'allument dans le paysage et dévoilent un décor à 
chaque fois nouveau, habité par des comédiens qui, en aucune manière, n'incarnent un personnage. Prenons une scène précise, illustrée par la photographie ci-dessous.

HoriZøne Km zéro, groupe ZUR

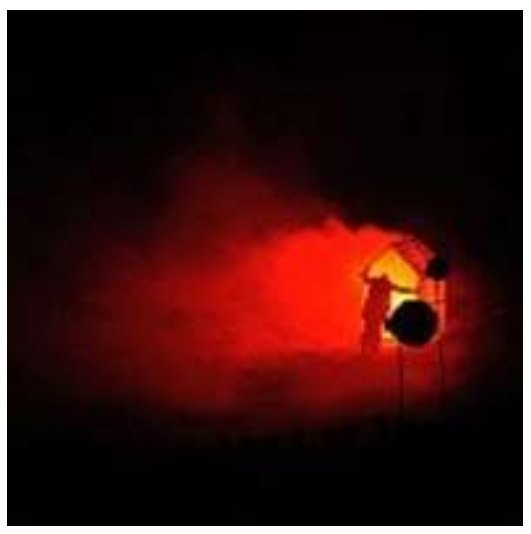

J-F Rabillon

L'entrée en scène ici a valeur d'apparition : quelqu'un ou quelque chose qui se manifeste, devient visible là où il n'y avait rien et qui, lorsque l'on s'en approche, disparaît. Les entrées en scène dans ce spectacle ont ainsi de manière presque systématique une valeur spectrale : elles travaillent le couple proche/lointain, premier plan / arrière plan, et dès que les spectateurs essaient de s'approcher de ces multiples scènes d'apparition, l'image s'évanouit.

14 Ainsi, ce spectacle, par ces entrées en scène répétées et travaillées dans une logique spectrale, réactive le fantasme du spectateur entrant au cœur du tableau. Le théâtre en extérieur, présenté dans de grands espaces naturels, joue de ce processus d'immersion fantasmatique, que l'entrée en scène permet d'activer.

\section{L'espace de la déambulation ou l'entrée perpétuelle}

15 La forme déambulatoire est en quelque sorte paradigmatique de l'idée que l'on se fait, de prime abord, du théâtre de rue : un spectacle mouvant, que l'on suit d'un bout à l'autre ou par morceaux, avec une attention discontinue de la part du public. Entrer en scène pour des comédiens de théâtre de rue dans une forme déambulatoire revient à s'immiscer dans l'espace quotidien des spectateurs : la forme déambulatoire fonctionne sur un continuum avec le monde réel et relève de la logique de l'effraction. Ni parenthèse ni bulle ni clôture, le temps de la performance est calqué sur le temps réel et vient en corrompre le cours.

La compagnie Komplex Kapharnaüm travaille pleinement ce paramètre de la mobilité, notamment dans son dernier spectacle intitulé Figures libres, qui thématisait l'entrée du théâtre dans l'espace du quotidien, aussi bien que l'entrée de langages quotidiens dans l'univers théâtral. Il y a peu de jeu d'acteurs dans les spectacles de cette compagnie, qui sont plus conçus comme des installations vidéos que comme des pièces à proprement parler. Ce sont donc des visages anonymes, des corps aux gestes quotidiens (se maquiller, mettre des boucles d'oreilles, etc.) qui « font leur entrée » sur les murs de la ville : ils sont projetés par un comédien installé sur un bus que les spectateurs suivent dans sa déambulation à travers la ville. Les visages projetés répètent sans cesse la question suivante : «Can you read my story on my face? » C'est en demandant des « histoires », en 
activant des dynamiques de récit, que le spectacle élabore un continuum entre corps des comédiens et corps des spectateurs. Ces spectateurs sont régulièrement invités à envoyer des messages sur un numéro de téléphone, destinés à être eux aussi projetés sur les murs de la ville. L'entrée en scène de multiples visages est ainsi un moyen de figurer l'entrée en scène de la foule anonyme qui suit la déambulation; les corps des spectateurs proposent un écho incarné aux corps projetés sur les murs.

\section{L'espace « éclaté mouvant ${ }^{9}$ »}

Dans les spectacles (Le) métalOrchestre / Virée(s) vers l'Est et Espèce H. - Mémoire vivante (2011), de la compagnie Metalovoice, les espaces de jeu sont éclatés et mobiles, et font reposer les entrées en scène des comédiens sur le mécanisme de la surprise, dans le but de créer un rapport de forces avec les spectateurs. Les comédiens, juchés sur d'imposants praticables sur roulettes, fendent la foule, l'obligeant à se redistribuer dans l'espace. Ces entrées successives s'accompagnent d'un jeu de lumière et de son, qui participe du mécanisme de la surprise. En effet, le spectacle se joue sur une place carrée ceinte de bâtiments, sur lesquels se réfléchissent les ombres des comédiens, qui font ainsi, d'une certaine façon, doublement leur entrée en scène. Les effets de lumière (les scènes mouvantes, éclairées, guident les spectateurs pour se répartir dans l'espace) participent ainsi pleinement de l'entrée en scène, qui s'assimile dans ce spectacle à une irruption. En effet, les jeux de lumière s'accompagnent d'un travail sonore principalement constitué de percussions sur d'immenses bidons d'usine, qui souligne la portée agressive de l'entrée en scène et métaphorise la violence politique contenue dans le spectacle. En proposant une scénographie aléatoire des entrées, les spectacles de la compagnie Metalovoice entendent jouer avec les attentes du spectateur et l'amener, précisément, à questionner sa place, son positionnement, tant théâtral que social.

\section{Sémiotique de l'entrée en scène}

\section{L'entrée et ses échos}

Dans certains spectacles, l'entrée en scène perd son caractère liminaire pour se démultiplier en écho tout au long du spectacle, sous la forme d'images fortes qui en cristalliseraient le sens profond. Dans Figures libres de Komplex Kapharnaüm, les différentes entrées en scène que l'on peut observer sont ainsi avant tout une réflexion sur ces figures qui donnent son titre au spectacle.

Komplex Kapharnaüm, Figures libres, 2012

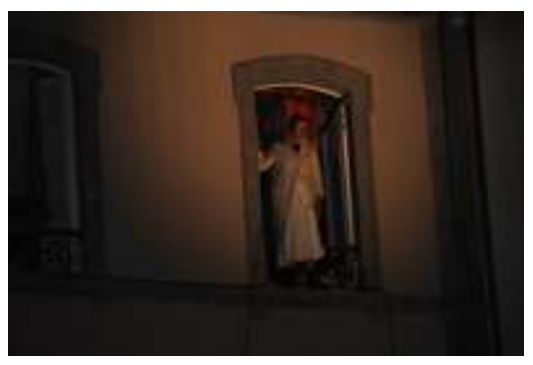

Fernand Prévot 
Komplex Kapharnaüm, Figures libres, 2012

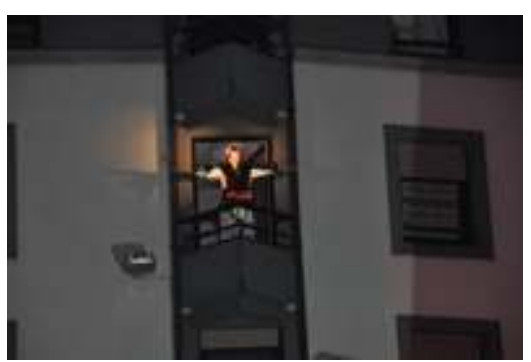

Fernand Prévot

Comme on peut le voir sur les deux photos ci-dessus, les apparitions de «figures » aux fenêtres des bâtiments de la ville, au fil du parcours déambulatoire, sont autant d'« effets d'entrée » qui rythment le spectacle, donnant l'occasion aux spectateurs de porter un regard inhabituel, décalé, sur la ville, métamorphosée par ces entrées en scène intempestives. Mais surtout, ces entrées plurielles questionnent dans un même mouvement la notion de "scène » et l'unicité de l'entrée en scène : dans ce spectacle, la scène est moins à proprement parler un espace qu'elle n'est définie par des cadres. Les entrées en scène s'y inscrivent dans une logique directement picturale où les délimitations spatiales servent un travail sémiotique: les différentes entrées visent à encadrer le sens du spectacle. Les apparitions aux fenêtres de la ville proposent autant de tableaux d'entrées, qui réécrivent le motif pictural de l'Annonciation: l'entrée de ces figures suspendues dans les airs s'assimile ainsi à une apparition dotée d'un caractère précisément "disruptif», si l'on reprend le terme de Denis Guénoun. Figures libres de Komplex Kapharnaüm repose sur l'alternance entre des moments de dilution du sens, notamment pendant la déambulation, et des moments de concentration sémiotique : les deux photographies que nous présentons ci-dessus montrent bien ces moments où le spectacle s'arrête, prend le temps de signifier, par le biais d'entrées en scène qui créent des images puissantes. Le spectacle se pense en continuité avec le réel, et la ville tout entière comme un gigantesque terrain de jeu. Chacun peut faire son entrée, et surgir d'un moment à l'autre, ce que font d'ailleurs beaucoup d'habitants qui voient passer la procession de leurs fenêtres : ils actualisent ainsi, une entrée quotidienne, que le spectacle programme.

Pour les Souffleurs et leur spectacle Pleine forêt sensible, l'entrée en scène, celle qui fait image, celle qui cristallise l'imaginaire du spectacle, est reportée. Elle n'a lieu qu'au milieu du spectacle, révélant en son centre son sens profond. 
Les Souffleurs, Pleine forêt sensible, 2012.

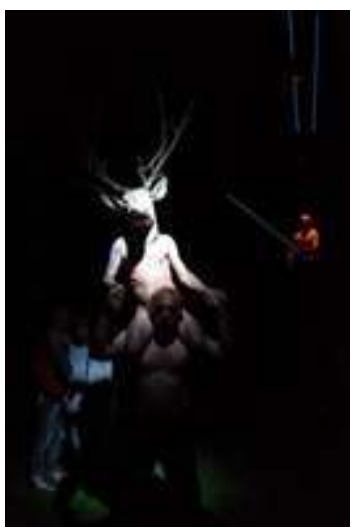

Christophe Raynaud de Lage

21 Cette entrée, qui revisite, réécrit, les différents types d'entrée qui l'ont précédée, réside dans une image qui captive les spectateurs et les oblige à reconsidérer leur répartition spatiale : un homme-cerf, porté sur les épaules d'un autre, entre en scène depuis un lieu mystérieux dans la forêt, et se fraye un passage, très lentement, parmi les spectateurs. À ce moment-là, de nombreuses idées esquissées pendant le spectacle prennent tout leur sens: l'entrée du cerf débride l'imaginaire du conte qui restait jusque là à l'état d'ébauche ; il marque un point d'aboutissement de tout le travail effectué précédemment par les comédiens avec les masques d'animaux de la forêt dont ils recouvraient régulièrement leur tête ; et, par sa charge symbolique et mythologique, l'image épaissit encore l'imaginaire foisonnant du spectacle et du lieu. On le voit sur la photographie, toutes les ressources techniques sont utilisées pour créer un " effet d'entrée " très fort, notamment la lumière. Les projecteurs dirigés sur le cerf, et uniquement sur lui, par contraste avec l'obscurité de la forêt, ne font qu'accentuer l'importance de cette entrée en scène.

\section{Entrée en scène et liminarité : l'entrée en scène élargie au rite}

La compagnie Kumulus, elle, a proposé avec Silence encombrant (2011) un spectacle qui n'est qu'une longue entrée en scène. La compagnie y interroge aussi des thèmes qui lui sont chers, comme l'exode, le questionnement identitaire, les questions de norme et de marge. Le spectacle, dont on voit le dispositif sur la photographie ci-dessous, travaille les mécanismes de l'entrée en scène sur deux plans. 


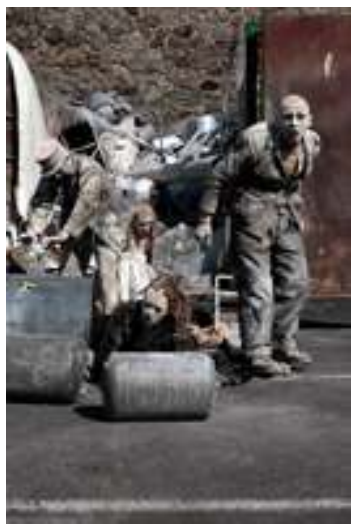

Vincent Muteau

Sur le plan thématique, le spectacle se propose de raconter, sans paroles, de manière purement visuelle, l'histoire d'individus aux marges de la société. En mettant en scène neuf comédiens qui sortent d'une benne à ordures, vêtus de vêtements abîmés et portant des objets vétustes, le spectacle réfléchit à la thématique de l'usage, appliquée tant aux êtres qu'aux objets. Recouverts de poussière blanche, les comédiens s'appliquent à donner aux spectateurs une image, un tableau d'entrée en scène: en ne proposant précisément qu'une longue entrée en scène théâtrale, le spectacle transpose esthétiquement des questionnements socio-politiques plus vastes.

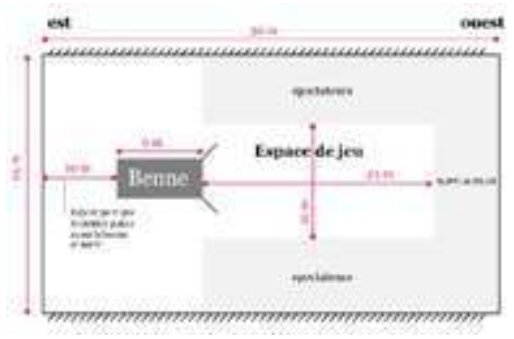

Schéma proposé par la compagnie Kumulus, dans la fiche technique du spectacle proposée sur leur site internet, http://www.kumulus.fr/repertoire/silence-encombrant.

Sur le plan scénographique, l'entrée en scène se fait dans un espace trifrontal comme le montre le schéma ci-dessus, mais l'espace des spectateurs n'est pas clairement délimité avant que le spectacle ne débute. En effet, les comédiens surgissent d'une benne à ordures jusqu'alors fermée, installée dans l'espace public et qui pourrait constituer un objet du quotidien. Les comédiens s'y sont installés bien avant que le public n'investisse les lieux et leur entrée en scène, sous la forme d'une irruption très lente hors de la benne, est précisément ce qui pousse les spectateurs à se disposer selon le dispositif tri-frontal que propose le schéma ci-dessus. Le spectacle dans son ensemble consiste en de lents et multiples allers-retours des comédiens, qui ne sont que des échos de l'entrée en scène initiale. Il s'agit de faire entrer toujours plus d'objets sur le plateau, de tirer de cette benne destinée aux ordures des objets usagés : dans le cadre de ce spectacle, l'entrée en scène permet de réfléchir à la notion de déjà-vu. En sortant du lieu par excellence du déchet et de l'usagé (la benne), les objets et les comédiens questionnent la capacité d'un spectacle à créer du nouveau, à se recycler; mais ce dispositif scénographique et dramaturgique donne également à l'entrée en scène dans ce spectacle la dimension d'un 
rite de passage au sens où l'entendait Victor Turner ${ }^{10}$. Ainsi, la « frontière » que constitue la porte de la benne, qui s'ouvre au tout début du spectacle incarne également un seuil rituel, seuil qui est à la fois l'objet même du rite et la métaphore des frontières en place dans le domaine du social. Bien sûr, ici les participants sont des comédiens, l'espace a été élaboré en amont, la benne disposée d'une certaine manière, les objets consciencieusement choisis ; néanmoins, le franchissement liminaire du seuil correspond bien ici, en un sens, à l'étape de la "marge » que Turner décrit comme partie prenante de tout rite de passage ${ }^{11}$. Si l'on postule que le rituel ne se situe pas dans un " autre monde ", dans un "ailleurs", mais interprète bien les rôles et les rapports sociaux existants, l'entrée en scène de ces «marginaux » de la compagnie Kumulus prend doublement la dimension d'un rite de passage: d'abord, toute entrée en scène théâtrale revêt une dimension rituelle dans la mesure où elle fait passer d'une situation sociale à une autre, et cela est d'autant plus vrai dans le contexte du théâtre de rue qui peut se rapprocher d'une logique carnavalesque ; ensuite, cette entrée en scène-là redouble, en exhibant des figures de marginaux, la seconde étape d'un rite de passage qui réside précisément en une forme de «marginalisation». Sans être pleinement de l'ordre du rite de passage, l'entrée en scène dans Silence encombrant peut être perçue comme une liminarité consciente, c'est-àdire une entre-deux rituel, dont les figures ne seraient ni ici, ni là, mais bien constamment sur un seuil entre la réalité sociale et la scène théâtrale.

Cette approche anthropologique de l'entrée en scène trouve un écho très fort si l'on prend en compte les participants du "rite »: acteurs et spectateurs, dans le cadre des théâtres de rue, semblent chacun proposer des formes d'entrées en scène, et activer ainsi la dimension fortement rituelle de la représentation/performance dans la mesure où, bien souvent, la limite entre ces deux pôles est largement perméable.

\section{Acteurs et spectateurs : interaction et inversion des rôles $^{12}$}

\section{Entrer avant l'entrée : l'exemple des Souffleurs, commando poétique}

Dans le spectacle des Souffleurs étudié plus haut, l'entrée des spectateurs dans l'espacetemps de la représentation est dissociée de l'entrée en scène des comédiens. Le spectacle a lieu, comme son titre l'indique, en pleine forêt, et l'on nous y emmène dans un bus affrété spécialement. Dès l'entrée dans ce bus, une femme annonce que «le spectacle commence ici ». Des boules Quiès, que l'on est invité à porter pendant le trajet en bus, sont distribuées pour créer une forme de sas de décompression, avant d'entrer dans le lieu secret de la représentation. Une fois arrivés, les spectateurs descendent du bus et sont guidés pour entrer dans la forêt par un marquage lumineux au sol qui les mène jusqu'à une clairière où les comédiens sont déjà en place. Il faut distinguer plusieurs niveaux dans ce protocole liminaire, qui constitue déjà une forme d'entrée en scène, si l'on en croit la définition de la performance, théorisée par Richard Schechner comme

la constellation de tous les événements, la plupart passant inaperçus, qui se produisent parmi les interprètes et les spectateurs entre le moment où le premier spectateur entre dans l'espace de jeu [...] et celui où le dernier spectateur sort ${ }^{13}$.

La notion de "performance ", dans le cadre des arts de la rue et particulièrement de ce spectacle, peut nous permettre de comprendre les différents niveaux d'analyse contenus 
dans la question de l'entrée en scène et semble plus juste concernant un spectacle qui refuse les modalités classiques de la représentation théâtrale.

Sur le plan scénographique, ce spectacle des Souffleurs invite à distinguer les instances qui entrent en scène. En effet, les spectateurs sont déjà entrés dans la sphère de la performance lorsqu'ils rencontrent les comédiens et ont été préparés à faire une expérience (sonore, visuelle) théâtrale. Pour la compagnie des Souffleurs, l'entrée en scène est un moment-clé et polyphonique : clé, parce que dans ce spectacle les instances sont inversées, ce sont les spectateurs qui entrent en scène, face à des comédiens déjà installés, déjà entrés; polyphonique, parce qu'elle prend en compte les voix des spectateurs (devenues chuchotantes en entrant dans la forêt) autant que celles des comédiens, soufflées dans les oreilles des spectateurs à l'aide d'un tube résonnant. L'entrée en scène des comédiens est complètement escamotée, en comparaison de celle des spectateurs : nous ne les voyons pas entrer, mais eux nous voient apparaître, selon un mécanisme d'inversion des rapports classiques regardants/ regardés. Faire entrer le spectateur est également un moyen de questionner, de remettre en jeu ce dispositif des regards. L'image liminaire du spectacle, dont on peut voir le dispositif ci-dessous, poursuit ce questionnement. L'évacuation de toute entrée en scène des comédiens trouble la frontière entre un avant et un après de la représentation, car on ne sait pas d'où ils parlent, d'où ils viennent. L'entrée en scène est aussi, avant tout, un questionnement de l'origine: l'escamoter, comme le font les Souffleurs dans ce spectacle, transforme les comédiens en êtres venus de nulle part.

Les Souffleurs, Pleine forêt sensible (2012).

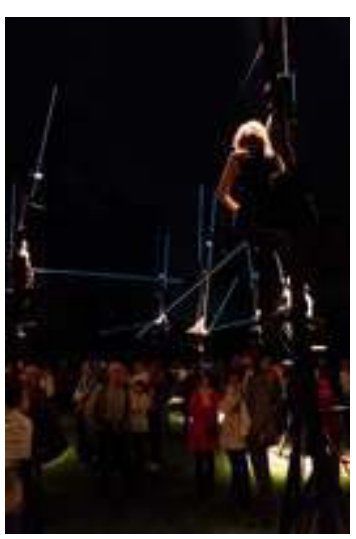

Christophe Raynaud de Lage

Sur le plan dramaturgique, ce spectacle des Souffleurs n'a de cesse de questionner les moyens d'entrer en scène, ou plutôt d'entrer dans la scène. En effet, le déroulement même du spectacle pousse le spectateur à s'inviter dans le jeu. Tous les spectateurs sont au milieu de ce dispositif scénique en pleine nature où les acteurs, suspendus dans les airs, dirigent leurs tubes poétiques vers tel ou tel spectateur pour lui souffler un poème à l'oreille. Cela crée parmi les membres du public une volonté de se frayer un chemin dans la foule et d'attraper un tube pour se créer son entrée en scène, pour faire partie pleinement du spectacle, et surtout, pour se créer un espace sonore propre, que personne d'autre n'entend. Dans ce spectacle des Souffleurs, l'entrée en scène du spectateur se fait sous la forme du secret. Ce qui lie le comédien et le spectateur est un secret : le spectacle particularise ainsi chaque membre du public. 


\section{L'entrée forcée : la convocation} l'interpelle, le met à l'épreuve, l'invite à monter sur scène. Souvent, le spectateur, choisi au hasard, est donc partie prenante du spectacle et se doit, lui aussi, de faire son entrée, et de la réussir. Ainsi, dans le spectacle Ma mort n'est la faute de personne (2012), de Nadège Prugnard et Marie-Do Fréval, quelques spectateurs, avant même que le spectacle ne soit réellement lancé, dans la file d'attente, se voient accostés par une femme qui leur demande : "Vous êtes bien Gildas? ». Certains jouent le jeu, d'autres non; mais dès que l'un se laisse prendre, la femme lui remet un petit carton, sur lequel on peut lire « Sachez que vous serez appelé par la morte. " Pendant le spectacle, on entend une des deux comédiennes dire : "On appelle le cousin Gildas » : le spectateur ainsi désigné se doit donc de faire son entrée, de s'allonger auprès de la morte, et de répondre à ses questions, qui ressemblent fort à des injonctions. L'entrée en scène du spectateur est ici une invitation à quitter l'indifférence, tant face au deuil (le thème du spectacle) qu'au théâtre lui-même, que l'auteure, Nadège Prugnard, entend bien secouer avec force. L'entrée en scène du spectateur est ainsi un moyen de faire tomber la frontière scène/salle, ce qui est confirmé par les adresses régulières d'une des deux comédiennes (qui est aussi la metteuse en scène) à l'ensemble des spectateurs (tour à tour invités à réciter ensemble une prière funèbre pour la morte, ou à allumer des bougies distribuées en début de spectacle). Le spectateur, qu'il soit appelé ou non sur la scène, est ainsi partie prenante du déroulement du spectacle, et son entrée en scène, collective ou individuelle, s'inscrit pleinement dans ce que Richard Schechner entend par "théatre participatif », et qui fait de l'entrée en scène du spectateur un «point de rupture » qui redéfinirait la place du théâtre dans le champ social.

31 La participation du public élargit le champ de la performance, parce que la participation du public se situe précisément au point de rupture où la performance devient un événement social. En d'autres termes, la participation est incompatible avec l'idée que l'œuvre d'art se suffit à elle-même, qu'elle est autonome, qu'elle a un début, un milieu et une $\mathrm{fin}^{14}$.

En ce sens, l'entrée en scène du spectateur va de pair avec une redéfinition plus ample des conditions mêmes d'exercice de l'art théâtral: en «désautonomisant » la scène, cette entrée revient à la fois sur la limite scène/assistance, et sur le déroulement linéaire et prévisible de l'acte théâtral. Les corps des spectateurs sont ainsi à prendre en compte comme capables à tout moment d'entrer en scène et de changer le cours du spectacle.

\section{Un seul corps?}

L'entrée en scène dans les arts de la rue permet donc une reconfiguration des instances de la représentation théâtrale : en se jouant des limites spatiales, elle propose de repenser l'opposition corporelle entre acteurs et spectateurs. Les arts de la rue postulent une scène globale, la scène de la ville, qui serait un terrain d'échange entre acteurs et spectateurs, et sur laquelle les spécificités de chacun seraient, en un sens, interchangeables. Si l'on en croit le point de vue de l'urbaniste et architecte Patrick Bouchain, les arts de la rue proposent une scène élargie à l'échelle de la ville entière : 
Dans la ville, le sol, c'est le niveau général réservé à l'échange. Jouer à cet endroit, c'est être sur le lieu même des opérations. Le cadre (de scène), c'est la ville, le plateau, c'est la rue. La dimension cachée du drame est dans la ville. La coulisse est la même pour le spectateur et l'acteur. C'est dans cet entre-deux que se réalise cette rencontre de plain-pied. C'est seulement tous ensemble qu'ils peuvent se libérer de leurs règles de distance; soudain, tout se passe comme s'il n'y avait qu'un seul corps [...]. Ce phénomène n'existe nulle part ailleurs. C'est l'endroit idéal pour les initiatives ${ }^{15}$. la part belle aux «initiatives » : tout un chacun, lié de près ou de loin au spectacle, est susceptible de surgir, de faire son entrée. Le spectateur du théâtre de rue est toujours un potentiel acteur, et inversement ; ces formes, par-delà leur diversité, se proposent toutes de rendre compatibles le cadrage et le décadrage. Elles créent des images encadrées dans un espace inattendu (comme on l'a analysé chez Komplex Kapharnaüm), images qui précisément sont là pour décadrer le regard des spectateurs et proposer une reconfiguration des instances quotidiennes.

\section{Conclusion}

Ce parcours à travers quelques spectacles de théâtre de rue conserve une part d'arbitraire: nombre de formes n'ont pas été abordées, dont les entrées en scène comportent d'autres spécificités. Néanmoins, l'étude des espaces a permis de constater les fluctuations des limites traditionnelles, mais aussi la diversité de ces formes, qui ne se réduisent pas à une table rase de toute délimitation. L'entrée en scène dans les arts de la rue se meut entre l'apparition et l'effraction, selon la logique de l'initiative, bien étudiée par Patrick Bouchain. L'initiative revêt un caractère premier : l'entrée en scène est bien de cet ordre dans la rue, elle qui, sans être nécessairement première temporellement, initie toujours un regard désaxé sur l'environnement dans lequel ce théâtre prend place.

Il nous faut enfin revenir sur la notion d'« entre-deux » que suggérait Patrick Bouchain, car elle semble très bien synthétiser les spécificités de l'entrée en scène dans les arts de la rue. En effet, cette entrée, plus que dans le théâtre dit "traditionnel », s'ancre dans un interstice qui revêt une densité rituelle très forte. Victor Turner, dans son étude du phénomène rituel, voit dans l'entre-deux l'espace-temps de la recombinaison des hiérarchies et statuts quotidiens, et en fait une caractéristique de la phase liminaire de tout rite de passage. L'entrée en scène, comme entre-deux, serait le lieu d'une ambiguïté comportementale («ni ici, ni là ») qui se développe dans un sens à la fois individuel et social.

Individuellement, l'entrée en scène dans la rue questionne la qualité d'acteur ou de spectateur, les deux étant potentiellement l'un et l'autre, dans une réversibilité proche de la logique carnavalesque. Socialement, cette entrée en scène est un questionnement de la frontière : si le théâtre en salle questionne également, à chaque entrée en scène, la valeur de la frontière entre la scène et le monde, le théâtre dans la rue redouble ce questionnement en faisant effraction dans l'espace social. Reste à se demander quelle peut être la valeur politique d'une telle effraction, et si cette entrée dans le champ social ne s'inscrit pas dans une logique rituelle qui en régulerait la valeur transgressive, ainsi que le suggère les analyses bourdieusiennes :

Le sens de la limite, qui sépare, et du sacré, qui est séparé, est indissociable du sens de la transgression réglée, donc légitime, de la limite qui est la forme par excellence 
$\mathrm{du}$ rituel : le principe de la mise en ordre du monde est encore au fondement des actions rituelles visant à rendre licites, en les déniant, les transgressions nécessaires ou inévitables des limites ${ }^{16}$.

\section{NOTES}

1. VITEZ Antoine, Antoine Vitez, «Du metteur en scène. De l'acteur.» Actes Sud Papiers, coll. « Mettre en scène ", Paris, 2006. [1988]

2. Voir notamment SCHECHNER Richard, Performance, expérimentation et théorie du théâtre aux USA, Paris, Editions théâtrales, 2008, et TURNER Victor, Le phénomène rituel, structure et contre-structure, Paris, PUF, 1990 [1969].

3. GONON Anne, Ethnographie du spectateur, le théâtre de rue, un dispositif communicationnel analyseur des formes et récits de la réception, 2007, p. 108 [en ligne] http://www.lefourneau.com/ ecritsmemoires/annegonon.pdf (page consultée le 7 décembre 2012).

4. Nous nous appuierons principalement sur les spectacles de trois compagnies: celle des Souffleurs, fondée en janvier 2001 par Olivier Comte, dont les spectacles sont composés notamment de poèmes faits pour être chuchotés à l'oreille des spectateurs à l'aide de cannes creuses en carbone ; la compagnie Kumulus, fondée en 1986 par Barthélémy Bompard, dont les spectacles traitent souvent de l'actualité sociale et de la représentation de catégories sociales peu représentées par ailleurs, parmi lesquels on peut citer à titre d'exemple Les Squames (1988), Itinéraires sans fond(s) (2003), Les Pendus (2009, co-écrit avec Nadège Prugnard) ; Metalovoice enfin, fondée en 1995, qui revendique le concept de "poésie industrielle » et dont le spectacle (Le) MétalOrchestre / Virée(s) vers l'Est (2011), abordait la thématique de la désindustrialisation et de ses conséquences.

5. Op. cit, p. 188.

6. GUENOUN Denis, «Scènes d'extérieur " Conférence-débat $n^{\circ} 1$. Scènes Invisibles, cycle de six conférences-débats sur les arts de la rue. Théâtre Paris-Villette, Paris, 30 janvier 2006. Cité par Anne Gonon, dans sa thèse, Ethnographie du spectateur. Le théâtre de rue, un dispositif communicationnel analyseur des formes et récits de la réception, www.lefourneau.com/ ecritsmemoires/annegonon.pdf [en ligne]. Page consultée le 25 janvier 2013.

7. Le Groupe ZUR (Zone Utopiquement Reconstituée) a été créé en 1984 et travaille notamment, dans ses spectacles, autour du caractère inattendu du lieu choisi pour la performance.

8. HorizØne, spectacle créé en 2009.

9. Nous reprenons là encore la terminologie d'Anne Gonon, op. cit, p. 108.

10. TURNER Victor, Le phénomène rituel, structure et contre-structure, Paris, PUF, 1990 [1969].

11. Selon Victor Turner, qui reprend les catégories élaborées au début du XXème siècle par Van Gennep, le rite comporte trois étapes: la séparation (étape pré-liminaire), la marge (étape liminaire), l'agrégation (étape post-liminaire). La « marge » ou « liminarité » se définit comme un espace-temps hors des normes sociales habituelles.

12. GONON Anne, Op. cit, p. 133 sqq. Elle identifie plusieurs types d'interactions acteurs / spectateurs: interaction structurelle, interaction visuelle, interaction d'adresse, interaction dialoguée, interaction d'implication. Dans le cadre de cette étude sur l'entrée en scène, c'est cette dernière qui retiendra notre attention. 
13. SCHECHNER Richard, Performance theory, Londres et New York, Routledge, 2003 [1988] ; cité par Anne Cuisset dans son introduction au premier chapitre de SCHECHNER Richard, Performance, expérimentation et théorie du théâtre aux USA, Paris, Editions théâtrales, 2008, p. 23.

14. SCHECHNER Richard, op. cit, p. 191.

15. BOUCHAIN Patrick, Intérieur Rue, Paris, Editions théâtrales, 2000, p. 58.

16. BOURDIEU Pierre, Le sens pratique, Paris, Editions de Minuit, 1980, p. 349.

INDEX

Mots-clés : théâtre de rue, spectateur, espace

\section{AUTEUR}

\section{GÉRALDINE PRÉVOT}

Doctorante à l'Université Paris X-Nanterre 